Research Paper

\title{
SDF-1 in Mammary Fibroblasts of Bovine with Mastitis Induces EMT and Inflammatory Response of Epithelial Cells
}

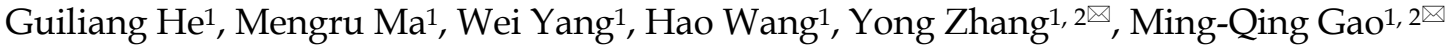 \\ 1. College of Veterinary Medicine, Northwest A\&F University, Yangling 712100, Shaanxi, China; \\ 2. Key Laboratory of Animal Biotechnology, Ministry of Agriculture, Northwest A\&F University, Yangling 712100, Shaanxi, China. \\ $\triangle$ Corresponding authors: College of Veterinary Medicine, Northwest A\&F University, Xinong Road 22, Yangling 712100, China. Tel: 86-29-87080092, Fax: \\ 86-29-87080085, E-mail:zhangyong1956@nwafu.edu.cn (Y. Zhang), or gaomingqing@nwafu.edu.cn (M.Q. Gao) \\ ( $)$ Ivyspring International Publisher. This is an open access article distributed under the terms of the Creative Commons Attribution (CC BY-NC) license \\ (https://creativecommons.org/licenses/by-nc/4.0/). See http://ivyspring.com/terms for full terms and conditions.
}

Received: 2017.02.09; Accepted: 2017.03.19; Published: 2017.05.05

\begin{abstract}
Fibroblasts constitute the majority of the stromal cells within bovine mammary gland, yet the functional contributions of these cells to mastitis and fibrosis and the mechanism are poorly understood. In this study, we demonstrate that inflammation-associated fibroblasts (INFs) extracted from bovine mammary glands with clinical mastitis had different expression pattern regarding to several extracellular matrix (ECM) proteins, chemokines and cytokines compared to normal fibroblasts (NFs) from dairy cows during lactation. The INFs induced epithelial-mesenchymal transition (EMT) and inflammatory responses of mammary epithelial cells in a vitro co-culture model. These functional contributions of INFs to normal epithelial cells were mediated through their ability to secrete stromal cell-derived factor 1 (SDF-1). SDF-1 was highly secreted/expressed by INFs, lipopolysaccharide (LPS) -treated NFs, lipoteichoic acid (LTA) -treated NFs, as well as mastitic tissue compared to their counterparts. Exogenous SDF-1 promoted EMT on epithelial cells through activating NF-KB pathway, induced inflammation response and inhibited proliferation of epithelial cells. In addition, SDF-1 was able to induce mastitis and slight fibrosis of mouse mammary gland, which was attenuated by a specific inhibitor of the receptor of SDF-1. Our findings indicate that stromal fibroblasts within mammary glands with mastitis contribute to EMT and inflammatory responses of epithelial cells through the secretion of SDF-1, which could result in the inflammation spread and fibrosis within mammary gland.
\end{abstract}

Key words: Stromal cell-derived factor 1, epithelial-mesenchymal transition, fibroblasts, epithelial cells, bovine mastitis.

\section{Introduction}

Bovine mastitis is the most important infectious disease of dairy cattle, affecting both the quality and quantity of milk produced in the world [1]. Bovine mastitis can cause varying degrees of fibrosis process. Current research suggests that the occurrence of tissue fibrosis is caused by exaggerated production of extracellular matrix (ECM) [2]. Activated fibroblasts with abnormal proliferation ability replace the damaged tissue and produce excessive ECM [3]. Epithelial-mesenchymal transition (EMT) is a process that epithelial cells undergo transition to a mesenchymal phenotype to produce fibroblasts and myofibroblasts. EMT has been taken as the potential sources of myofibroblasts in tissue fibrosis [2].

Bovine mammary fibroblasts are main stromal cells of the mammary gland that regulate epithelial cell behavior through direct and indirect cell-cell interactions. Stromal fibroblasts also lead to persistent inflammation of the mammary gland by secreting cytokines, chemokines and growth factors [4]. Fibroblasts are important sentinel cells in the immune system, and it is proposed that these cells play a 
critical role in the switch from acute inflammation to adaptive immunity and tissue repair [5]. There is now abundant evidence that fibroblasts taken from diseased tissues display a fundamentally different phenotype compared with fibroblasts taken from normal tissues at the same anatomical site [6, 7]. As fibroblasts are relatively long-lived cells, a tight control mechanism must exist to prevent overstimulation of the immune system, which would lead to persistent inflammation [6-8]. The molecular basis for the persistent, activated phenotype of fibroblasts at sites of chronic inflammation remains unclear [5].

Stromal cell-derived factor 1 (SDF-1), also known as CXCL12, is a small cytokine belonging to the chemokine family and originally described as a chemoattractant for lymphocytes $[9,10]$. In chronic inflammation, tissue fibroblasts fail to switch-off production of SDF-1, leading to the sustained and inappropriate retention of lymphocytes as well as dendritic cells within the inflamed tissue. In vitro studies revealed that SDF-1 was secreted by cancer associated fibroblasts or myofibroblasts and bound to its receptors (CXCR4 and CXCR7) on cancer cell surface to activate downstream intracellular signal pathways that regulate the metastasis, angiogenesis, and drug-resistant of cancer cells[11]. Kang et al overexpressed SDF-1 in MDA-MB-231 cells to create an autocrine loop of SDF-1/CXCR4 and found that SDF-1 boosted the invasiveness and migration of breast cancer cells [12]. SDF-1 activates a variety of intracellular signal transduction pathways regulating in cell migration, invasion, survival, proliferation, and adhesion $[1,13,14]$. Although SDF-1 is crucial for the migration and invasion of cells, little is known about the roles of SDF-1 in induction of EMT and inflammatory response in bovine mammary epithelial cells.

In the present study, we sought to elucidate the properties of fibroblasts isolated from bovine mammary tissue with mastitis that facilitate the spread of inflammation and fibrosis within the mammary gland.

\section{Materials and Methods}

\section{Cell culture}

Inflammation-associated fibroblasts (INFs) extracted from bovine mammary glands with clinical mastitis, normal fibroblasts (NFs) from slaughtered dairy cows because of fractured legs during lactation, and normal epithelial cells from normal bovine mammary tissue used in this study were isolated by our experiment team in previous research [15]. Cells were recovered and cultured in DMEM/F12 containing $10 \%$ fetal bovine serum (FBS) and 100 $\mathrm{IU} / \mathrm{ml}$ penicillin with $100 \mu \mathrm{g} / \mathrm{ml}$ streptomycin (Gibco BRL, Grand island, NY), respectively, and cultured at $37^{\circ} \mathrm{C}$ in a humidified $5 \% \mathrm{CO}_{2}$ environment. Each type of cells was used as cell pools by mixing equivalent amounts of cells from all individuals in all experiments. To retain the in vivo properties of isolated fibroblasts, we used in vitro culture fibroblasts within the fifth passage after they were extracted from the mammary tissue.

\section{Cell treatment}

Inflammatory responses of NFs to Escherichia coli and Staphylococcus aureus were induced by their cell wall components lipopolysaccharide (LPS) and lipoteichoic acid (LTA), respectively. Before stimulation with LPS(Sigma-Aldrich, St Louis, MO) and LTA (InvivoGen, San Diego, CA), confluent NFs were cultured in minimum serum medium (MSM) composed of DMEM/F12, 0.5\% FBS and antibiotics for $24 \mathrm{~h}$ at $37^{\circ} \mathrm{C}$. Then, the medium was replaced with complete medium containing LPS or LTA at final concentrations of $10 \mathrm{ng} / \mu \mathrm{l}$ for $3 \mathrm{~h}$ or $20 \mathrm{ng} / \mu \mathrm{l}$ for $12 \mathrm{~h}$ according to recommended concentrations and treatment periods in a previous publication[16] in which an in vitro inflammatory cell model of mastitis induced by LPS or LTA was established. After stimulation, cell culture supernatants were discarded and replaced with MSM. The culture was incubated for another $24 \mathrm{~h}$. Finally, cell culture supernatants were collected for IL-8, CCL5 and CXCL2 secretion measurement by ELISA, and cells were harvested for protein extraction and western blot analysis of a-SMA, collagen-1 and vimentin. NFs cultured in MSM and without stimulation were used as controls.

\section{Indirect co-culture in vitro}

The effects of NFs and INFs on epithelial cells were investigated through an indirect co-culture model by using conditioned medium (CM). In brief, NFs or INFs were rinsed with PBS. Then fresh serum-free DMEM/F12 medium was added to the culture dishes and cultured for another $24 \mathrm{~h}$. Cell culture supernatant was collected and used as CM by mixture with complete medium at a 9:1 ratio. Finally, cell culture supernatants were collected and epithelial cells were harvested for subsequent analysis after 3-day culture in CM. Epithelial cells cultured in MSM were taken as control.

To investigate whether inflammatory stromal fibroblasts induced by LPS or LTA could promote SDF-1-mediated inflammatory response in epithelial cells, epithelial cells were cultured in CM from LPS- or LTA-treated NFs. Firstly, CM was collected from NFs that were pre-treated by LPS or LTA as 
aforementioned. And epithelial cells were pretreated by AMD3100 (MedChem Express, Princeton, NJ), a specific inhibitor of the receptor of SDF-1, for $24 \mathrm{~h}$ at the concentration of $25 \mu \mathrm{g} / \mathrm{ml}$. Then, AMD3100-pre-treated and untreated epithelial cells were cultured in CM from LPS- or LTA-treated NFs. Finally, cell culture supernatants were collected to measure the secretion TNF- $\alpha$ at day 3 after culture using an ELISA Kit. Epithelial cells cultured in MSM were used as control.

\section{ELISA}

For detection of IL-8, CCL5, CXCL2 and SDF-1 secreted by NFs or INFs, cells were cultured and grown to around $80 \%$ confluence. The medium was replaced with MSM, and then cultured for another 48 $h$. The media were finally collected and centrifuged to remove the cell debris. For detection of TNF- $\alpha$ secreted by epithelial cells induced by SDF-1 treatment, the cells were treated with exogenous SDF-1 at final concentration of $0,20,40$, and $60 \mathrm{ng} / \mathrm{ml}$ for $24 \mathrm{~h}$, and the medium was replaced with MSM and continued to culture for $24 \mathrm{~h}$. Cell culture supernatants were mixed to detect the secretion of TNF-a. The amount of IL-8, CCL5, CXCL2, SDF-1 and TNF- $\alpha$ in the medium was measured with corresponding immunoassay kits (Huzhen Biological Technology, Shanghai, China) in accordance with the manufacturer's instructions.

\section{Western blot analysis}

Cells were cultured and grown to around 80\% confluence. Cells in each sample were lysed with PRO-PREP Protein Extraction Solution (iNtRON Biotechnology, Inc. Gyeonggi-do, Korea). For NF-kB p65 detection, nuclear and cytoplasmic fraction proteins were extracted using Nuclear and Cytoplasmic Protein Extraction kit (Beyotime, Shanghai, China) following the manufacturer's instructions. All protein concentrations were detected using a Bradford protein assay kit (TransGene, Beijing, China). Then equal amount of protein for each sample was loaded and separated using SDS-polyacrylamide gel electrophoresis and electrotransferred onto polyvinylidene fluoride membranes (Millipore, Bedford, MA). After being blocked with $10 \%$ non-fat milk for $2 \mathrm{~h}$, the membranes were probed with primary antibodies against MMP-1, collagen-1, vimentin (all 1:1000 diluted, Bioss, Beijing, China), Cytokeratin (1:1000 diluted, Sangon, Shanghai, China), p65 (1:500 diluted, Santa Cruz, Dallas, TX), p-p65 (1:500 diluted, Bioss), GAPDH (1:1000 diluted, TransGen) at $4^{\circ} \mathrm{C}$ overnight and subsequently incubated with their corresponding secondary antibodies (1:2000 diluted, Beyotime) for 2 $\mathrm{h}$ at $37^{\circ} \mathrm{C}$.Unbound antibodies in each step were washed three times with TBST, per time ten minutes. The positive bands were visualized through enhanced chemiluminescence solution (Beyotime). GAPDH served as internal controls

\section{RNA extraction and real-time quantitative PCR (RT-qPCR) analysis}

Tissue samples were repeatedly grinded to a fine powder with liquid nitrogen using a mortar and pestle, and then the sample was transferred to $1.5 \mathrm{ml}$ non-enzyme EP tube. All samples were respectively resuspended in ice-cold TriZol solution (TransGene); samples were passed through aribonuclease-free 20-gauge needle 10-20 times for disruption. Total mRNA extraction was performed with the RNA Easy Kit (TransGene) according to the manufacturer's instructions, and concentration was measured with a spectrophotometer (BioTek, Winooski, VT), and 1000 ng of RNA was reverse-transcribed into cDNA with the TransScript II First-Strand cDNA Synthesis Super Mix (TransGene).

All PCR primers were synthesized by Sangon Biotech (Shanghai, China). The primers are listed in Supplementary Table S1. RT-qPCR was done on ABI Step One Software System (ABI, Foster City, CA). PCR conditions were as follows: $95^{\circ} \mathrm{C}$ for 30 seconds, then 40 cycles of denaturation at $95^{\circ} \mathrm{C}$ for 5 seconds, and finally at $60^{\circ} \mathrm{C}$ for 30 seconds. Primer specificity was estimated by melting curves. Standard curves were generated with reference cDNA to determine the starting quantity of mRNA in all samples. Comparative $\mathrm{Ct}$ method was employed to quantify normalized specific gene expression relative to the calibrator. Data were expressed as relative gene expression $=2-\Delta \Delta \mathrm{Ct}$, and GAPDH served as an internal control.

\section{Cell proliferation}

The proliferation of the cells was detected by using a CCK-8 kit (Beyotime). Briefly, $100 \mu \mathrm{l}$ of cell suspension containing 1000 cells were dispensed in quadruple in 96-well microplates and cultured for 24 h. Then, the cells were stimulated with SDF-1 (Peprotech, RockyHill, NJ) at a final concentration of 0, 20, 40 or $60 \mathrm{ng} / \mathrm{ml}$. After incubation for indicated time periods, the medium was replaced with $100 \mu \mathrm{l}$ fresh medium containing $10 \mu \mathrm{l}$ of CCK-8 solution and incubated for an additional $3 \mathrm{~h}$ at $37{ }^{\circ} \mathrm{C}$ in a humidified incubator. The optical density of each well of 96-well plates at $450 \mathrm{~nm}$ wavelength was measured in a microplate reader (BioTek). Cell proliferation rate was calculated by the formula $\left[\left(\mathrm{A}_{\mathrm{n}}-\mathrm{A}_{\text {blank }}\right)-\left(\mathrm{A}_{0}-\right.\right.$ $\left.\left.A_{\text {blank }}\right)\right] /\left(A_{0}-A_{\text {blank }}\right) \times 100$, where $A_{n}$ is the absorbance value at the indicated time of $24 \mathrm{~h}, 48 \mathrm{~h}$ and $72 \mathrm{~h}$ after 
culture, $A_{0}$ is the absorbance value at $0 \mathrm{~h}$, and $A_{\text {blank }}$ is the absorbance value of the wells without cells.

\section{Inflammatory induction studies in mice}

Female Kunming strain mice at 2-3 months old were obtained from Laboratory Animal Center of Fourth Military Medical University (Xi'an, Shaanxi, China) and maintained in accordance with the institutional guidelines of Northwest A\&F University. All animal studies were carried out according to experimental protocols approved by the Animal Care Commission of the college of Veterinary Medicine, Northwest A\&F University. All the animals obtained human care, and the study was executed in accordance with the institution's guidelines.

The lactating mice, 5-7 days after birth of the offspring, were randomly divided into three groups: the blank control group, SDF-1 group and SDF-1 + AMD3100 group. The mice were anesthetized by pentobarbital sodium. A $1000-\mu l$ syringe with a 30-gauge blunt needle was used to inoculate both L4 (on the left) and R4 (on the right) abdominal mammary glands. The anesthetized mice were placed on their backs under a binocular microscope. The teats and the surrounding area were disinfected with $70 \%$ ethanol. Each udder canal was exposed by a small cut at the near end of the teat and then challenged via teat

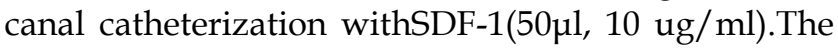
mice in the blank control group were given $50 \mu \mathrm{l}$ of normal saline. AMD3100 ( $50 \mu \mathrm{l}, 0.5 \mathrm{mg} / \mathrm{ml})$ was given by intraperitoneal injection in advance for $24 \mathrm{~h}$ of SDF-1 administration in accordance with previous studies [17]. At $24 \mathrm{~h}$ after infusion, the mice were killed using cervical dislocation and a part of mammary tissues were collected and fixed in formalin and embedded in paraffin and then stained with hematoxylin and eosin for routine histopathological analysis.

\section{Statistical analysis}

The results are expressed as means \pm standard deviation (SD). All statistical analyses were performed using ANOVA with the Bonferroni post hoc test. (SPSS 11.5, IBM Corporation, Armonk, NY). $\mathrm{P}<0.05$ was considered statistically significant.

\section{Results}

\section{INFs showed different expression pattern compared to NFs regarding to several ECM proteins, chemokines and cytokines}

Numerous studies suggest that fibroblasts taken from diseased tissues have a fundamentally different phenotype compared with fibroblasts taken from normal tissues at the same anatomical site. Fibroblasts are ubiquitous cells that provide mechanical strength to tissues by providing as supporting framework of ECM [5]. To investigate whether NFs and INFs exist the expression differences of ECM proteins. The expression mRNA and protein levels of collagen-1, vimentin, and MMP1 in NFs and INFs were analyzed by RT-PCR and western blot, respectively. The results showed that INFs expressed higher levels of vimentin and collagen-1than NFs, but INFs expressed a lower level of MMP-1 than NFs in both mRNA and protein level (Figure 1A and B). In addition, fibroblasts also react to persistent inflammation of the mammary gland by secreting cytokines, chemokines and growth factors [5]. To investigate the secretion of cytokines and chemokines by INFs and NFs, we detected the expression of IL-8, CXCL2, and SDF-1 in mRNA level by RT-PCR and the secretion of IL-8, CXCL2 and SDF-1 in protein level by ELISA. We found that INFs expressed higher mRNA and secreted higher protein of IL-8, CXCL2 and SDF-1 compared with NFs (Figure $1 C$ and $D)$. This finding suggested that mammary stromal fibroblasts within mammary glands after infection may contribute to the fibrosis by increased deposition of ECM protein in stromal, and have profound effects on epithelial cells by accumulated cytokines and chemokines

\section{The inductive expression of cytokines, chemokines and ECM proteins in fibroblasts by LPS and LTA in vitro}

A mastitis cell model has been established with LPS- and LTA-treated epithelial cells in our previous study [16]. To validate whether the cell wall components LPS and LTA of Escherichia coli and Staphylococcus aureus were able to cause the elevated expression of these examined cytokines, chemokines and ECM proteins in INFs, NFs were incubated with LPS or LTA, and IL-8, CXCL2 and CCL5 secretion was quantified by ELISA. The results showed a strongly enhanced output of IL-8, CXCL2 and CCL5 by NFs after LPS or LTA induction compared to NFs without stimulation (Figure 2A). In addition, the expressions of vimentin, collagen-1, and a-SMA were analyzed by western blot, and the results showed that the protein of collagen-1, vimintin and a-SMA had a significant increase in LPS- or LTA-treated NFs compared to untreated NFs (Figure 2B).

\section{INFs promote EMT in mammary epithelial cells}

In order to study whether the fibroblasts isolated from mammary glands with mastitis have biological effects on epithelial cells, normal epithelial cells were co-cultured with INFs and NFs in vitro in an indirect co-culture model by using conditioned medium. We found that the epithelial cells had morphological 
changes from cobblestone-like to spindle-like shape after they were co-cultured with both NFs and INFs, but this change was more significant in cells co-cultured with INFs compared to NFs (Figure 3A). We further detected the expressions of two well-known EMT markers, cytokeratin and a-SMA, in epithelial cells. INFs up-regulated a-SMA and down-regulated cytokeratin expression in epithelial cells in both mRNA and protein level (Figure 3B and C) compared to NFs, indicating stromal fibroblasts extracted from mammary gland with mastitis have an induction effect of EMT to epithelial cells.

\section{SDF-1 is involved in INFs-induced EMT in epithelial cells through NF-KB pathway}

It was reported that SDF-1 could trigger EMT of breast cancer cells through NF-kB pathway. We have confirmed that INFs expressed higher level of SDF-1 than NFs (Figure 1C and D). The expression of SDF-1 in tissue of mammary glands with or without mastitis was also analyzed, and the result of real time PCR showed that mastitic tissue had a higher SDF-1 expression than normal tissue (Figure 4). In addition, LPS or LTA treatment induced an increased expression of SDF-1 mRNA (Figure 5A) and protein level (Figure 5B) in stromal fibroblasts.
A

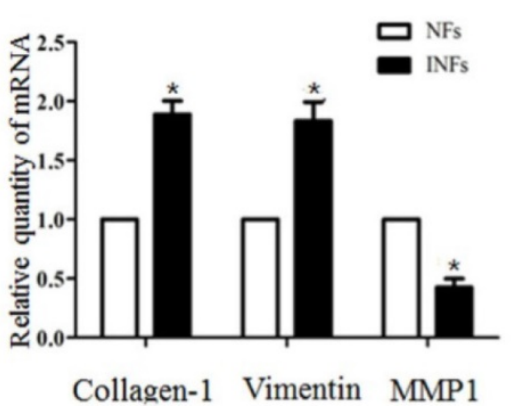

$\mathrm{C}$

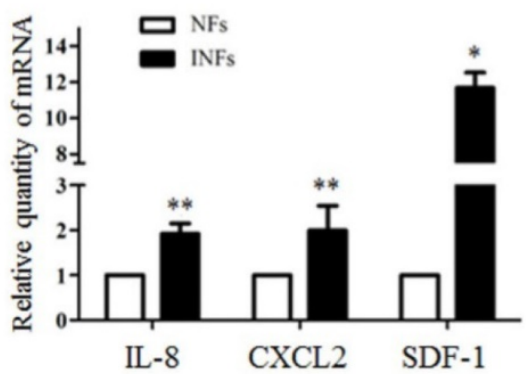

B

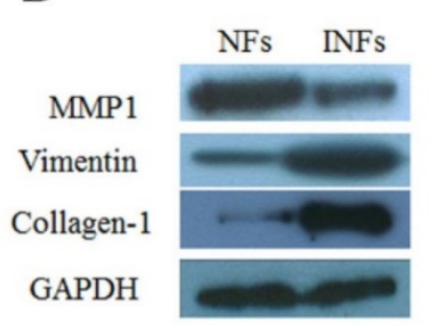

D

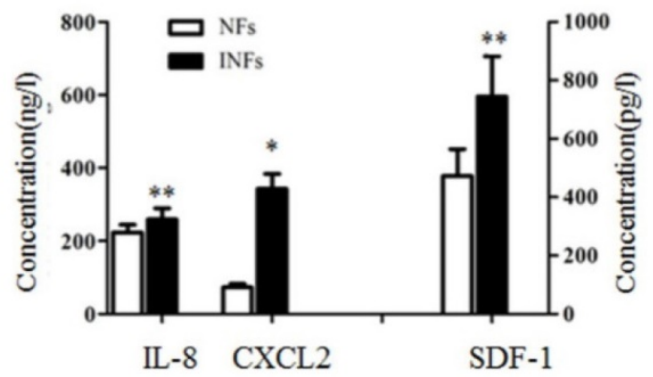

Figure 1. INFs showed difference expression pattern compared to NFs regarding to several ECM proteins chemokines and cytokines. (A) Gene expressions of collagen-1, vimentin, and MMPI in NFs and INFs analyzed by RT-qPCR. GAPDH was used as an internal control.*P<0.01 vs NFs. (B)The representative images of collagen-1, vimentin, and MMPI expression in NFs and INFs analyzed by Western blot. The quantitative analysis of each protein was relative to GAPDH. $* P<0.01$ vs NFs. (C) Gene expressions of IL-8, CXCL2, and SDF-1 in NFs and INFs were analyzed by RT-qPCR. GAPDH was used as an internal control. *P<0.01, $* * \mathrm{P}<0.05$ vs NFs. (D) Protein secretion of IL-8, CXCL2, and SDF-1 by NFs and INFs measured by ELISA. *P<0.01, **P $<0.05$ vs NFs. All results are given as mean \pm SD, and the error bars represent the SD from three independent experiments.
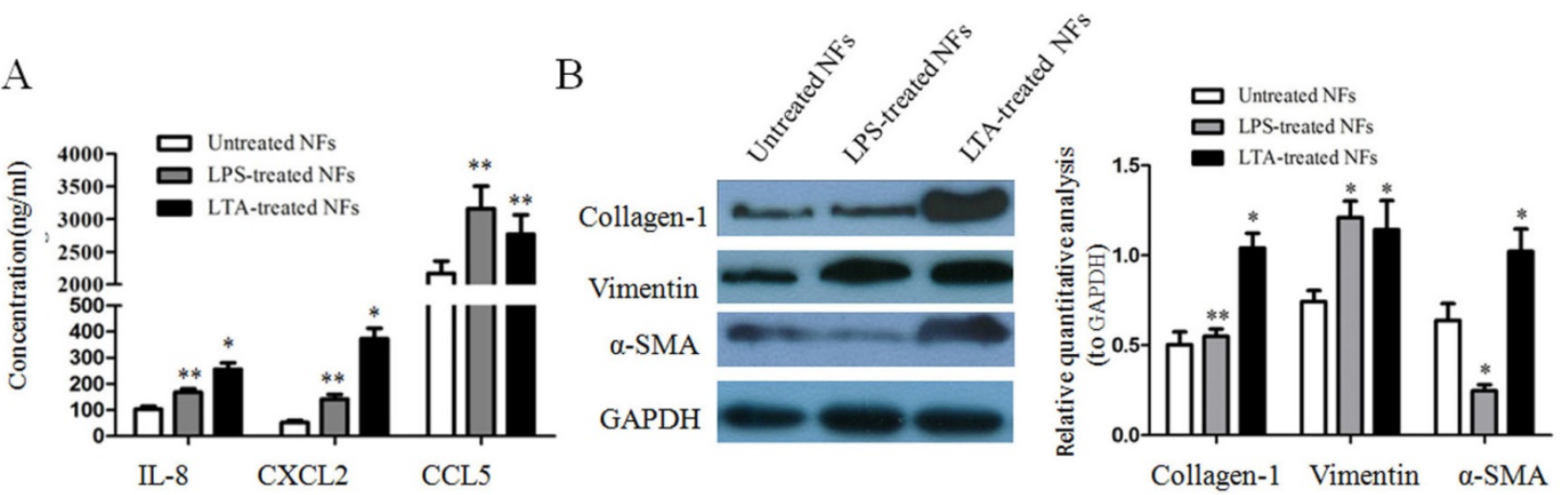

Figure 2. The inductive expression of cytokines, chemokines and ECM proteins by LPS and LTA in vitro. (A) The concentration of IL-8, CCL5 and CXCL2 in the medium secreted by NFs treated or untreated with LPS or LTA was measured by ELISA. $* P<0.01$, $* * P<0.05$ vs untreated NFs. (B) Representative blots of $\alpha$-SMA, collagen-1 and vimentin in NFs treated or untreated with LPS or LTA were analyzed by western blot. GAPDH was used as internal controls. $* P<0.01$, **P $<0.05$ vs untreated NFs. The above results are given as mean $\pm \mathrm{SD}$, and the error bars represent the SD from three independent experiments. 
A
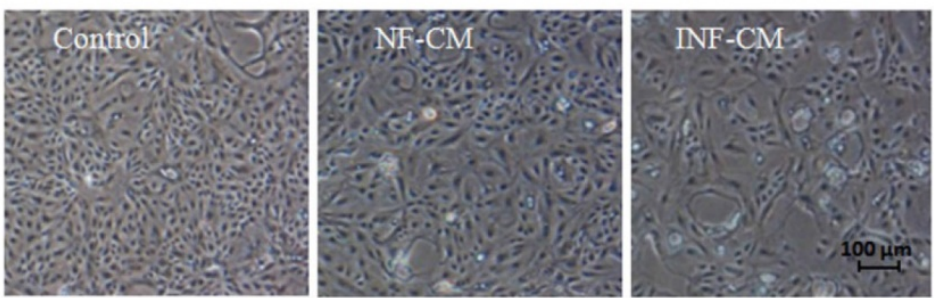

$\mathrm{B}$

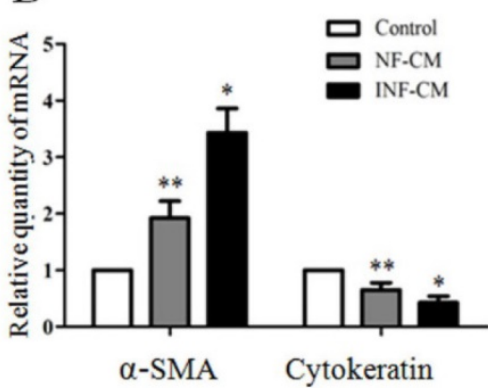

$\mathrm{C}$

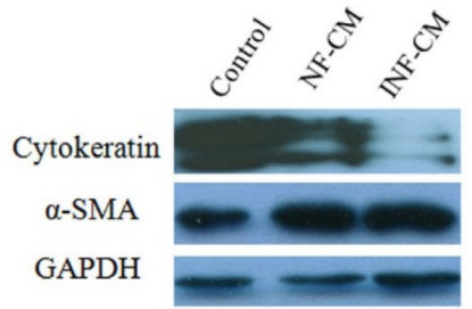

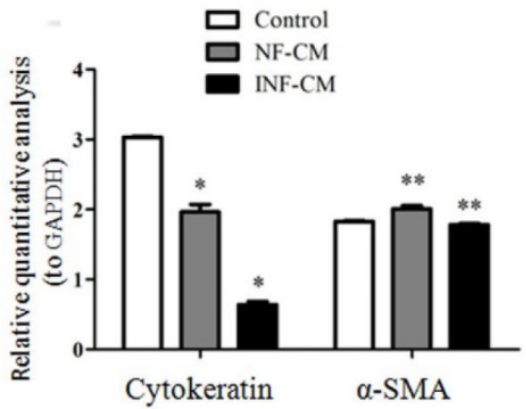

Figure 3. INFs promote EMT in mammary epithelial cells. (A) Morphological changes of epithelial cells induced by NFs-condition medium (CM) and INFs-CM. The majority of epithelial cells cultured in INF-CM developed a spindle fibroblast-like morphology, while cells cultured in NF-CM or minimum serum medium (MSM, Control) maintained the typical epithelial cobblestone pattern. (B) Gene and (C) protein expression changes of $\alpha$-SMA and cytokeratin in epithelial cells analyzed by RT-qPCR and western blot, respectively. GAPDH was used as internal controls. Expression of $\alpha$-SMA at both mRNA and protein level were increased in epithelial cells cultured in INF-CM to a greater extent than those cultured in NF-CM or Control, while INF-CM downregulated cytokeratin expression in epithelial cells at both mRNA and protein level compared to NF-CM and Control. $* \mathrm{P}<0.01, * * \mathrm{P}<0.05$ vs NF-CM and Control. The above results are given as mean \pm SD, and the error bars represent the SD from three independent experiments

A

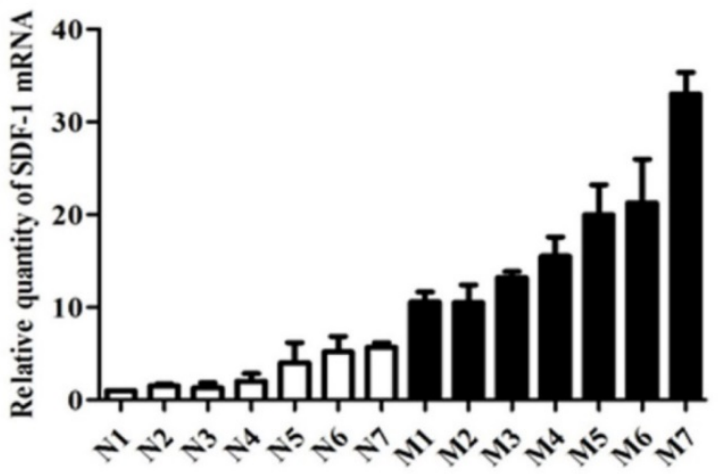

$\mathrm{B}$

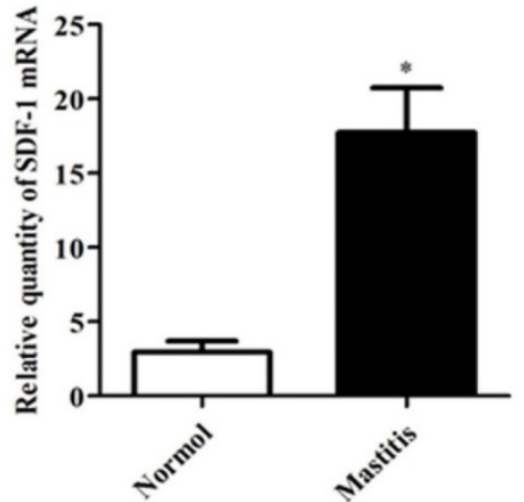

Figure 4. The expression of SDF-1 in mammary tissue of bovine with or without mastitis. (A) The mRNA expression of SDF-1 in normal ( $n=7$ ) and mastitic $(n=7)$ tissue of mammary glands analyzed by RT-qPCR analysis. GAPDH was used as an internal control. The results are given as mean \pm SD, and the data were from three independent experiments. (B) Statistic analysis of the expression of SDF-1 between normal group and mastitis group. $* \mathrm{P}<0.01$ vs Normal.
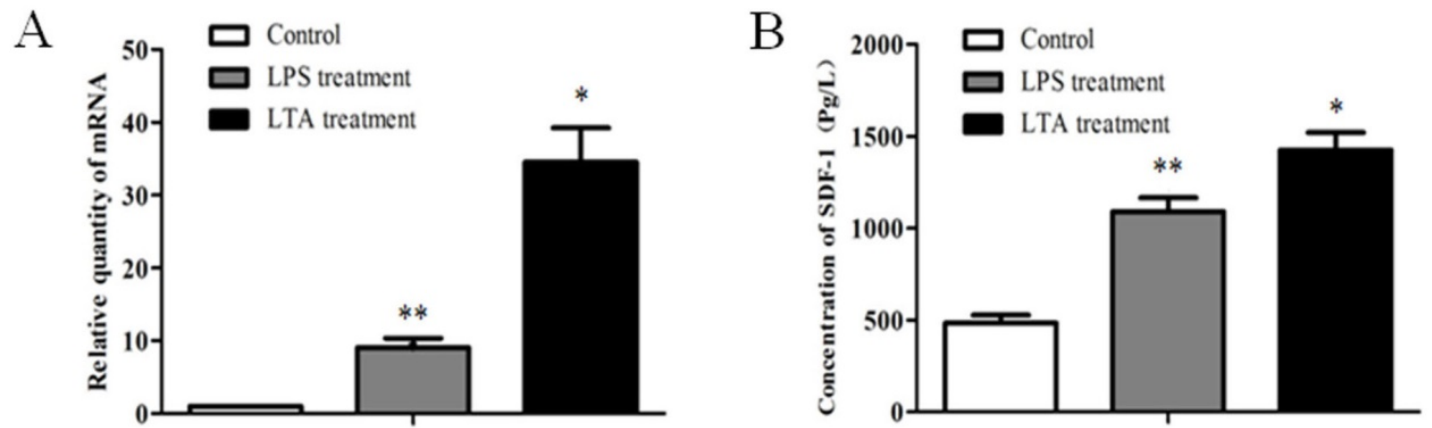

Figure 5. The expression of SDF-1 in stromal fibroblasts stimulated by LPS and LTA. (A)The mRNA expression of SDF-1 in LPS and LTA-treated fibroblasts analyzed by RT-qPCR. (B) The concentration of SDF-1 in medium secreted by LPS- and LTA -treated fibroblasts measured by ELISA. NFs without treatment were used as control. Both LPS and LTA enhanced the expression of SDF-1 at mRNA and protein level in stromal fibroblasts compared to control. $* \mathrm{P}<0.01, * * \mathrm{P}<0.05$ vs control. The above results are given as mean $\pm \mathrm{SD}$, and the error bars represent the SD from three independent experiments 
In order to further validate the whether increased SDF-1 in stromal fibroblast and mammary tissue with mastitis could cause EMT of epithelial cells, exogenous SDF-1 was used to treat mammary epithelial cells, and morphological changes and expression levels of EMT-related proteins in epithelial cells were examined. At $24 \mathrm{~h}$ after treatment with SDF-1, obvious morphological changes of epithelial cells from cobblestone-like to spindle-like shape were observed at the concentration of 40 and $60 \mathrm{ng} / \mathrm{ml}$ (Figure 6A).We further detected the EMT protein markers of a-SMA, vimentin and cytokeratin by western blot, and the results showed that SDF-1 up-regulated the expressions of a-SMA and vimentin down-regulated the expression of cytokeratin (Figure 6B). All this indicated that SDF-1 was able to trigger EMT of epithelial cells.

To investigate the mechanism by which SDF-1 induced EMT in epithelial cells, western blot analysis was applied to examine the expression levels of crucial molecular proteins of NF-kB pathway. As shown in (Figure 6C), the level of p65 phosphorylation was significantly increased in epithelial cell after treatment with SDF-1 compared to control. In addition, we also observed an obvious decrease of cytoplasmic p65 level and a significant increase of nuclear p65 level in epithelial cells treated with SDF-1 compared with control (Figure 6D).
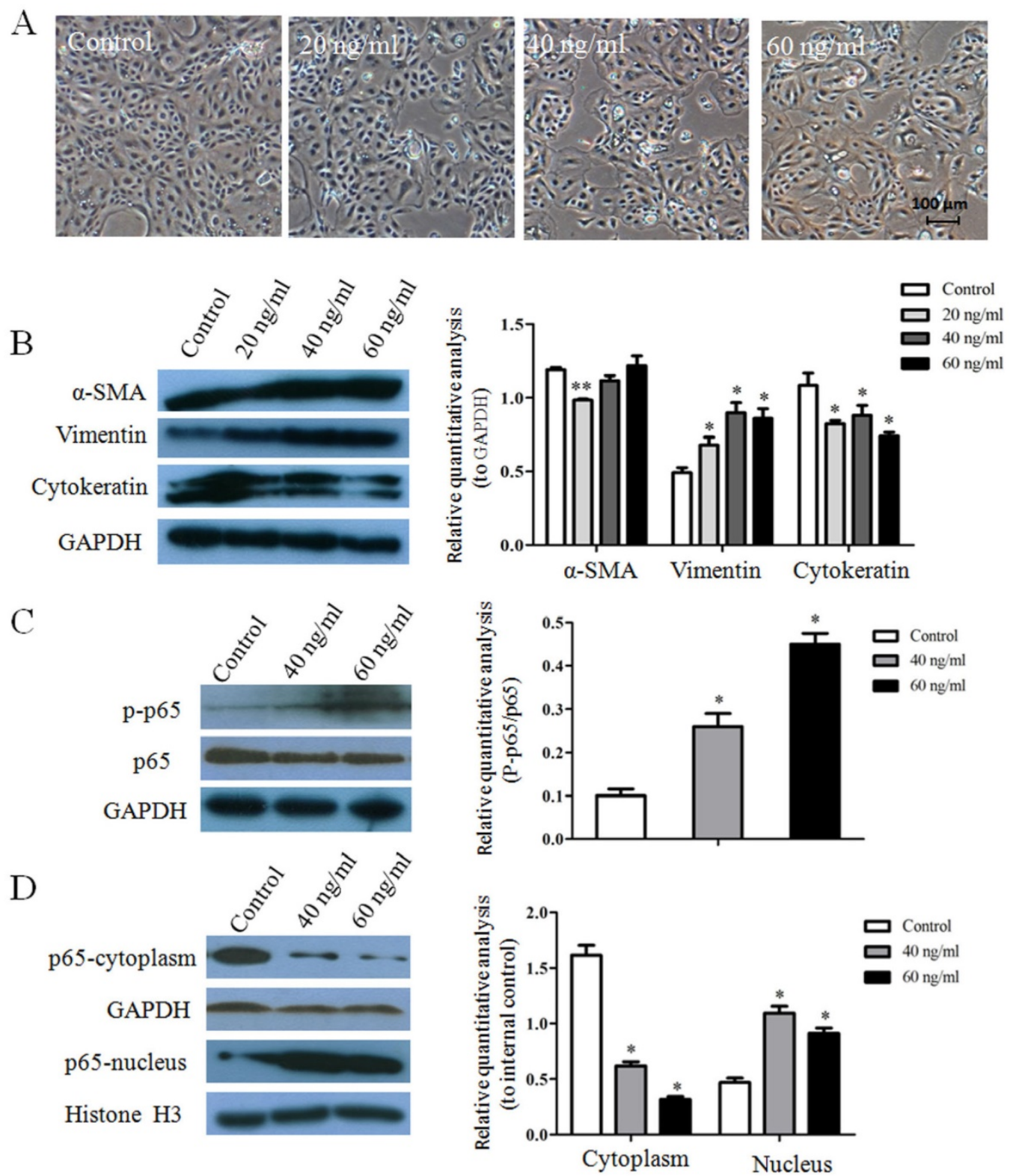

Figure 6. SDF-1 is involved in INFs-induced EMT in epithelial cells through NF-KB pathway. (A) Morphological changes of epithelial cells stimulated by SDF-1 at the concentration of 0 (control), 20,40 , and $60 \mathrm{ng} / \mathrm{ml}$. (B) Representative blots of $\alpha$-SMA, vimentin and cytokeratin and (C) P-p65 and p65 in epithelial cells stimulated by SDF-1 analyzed by western blot. The quantitative analysis of each protein was relative to internal control. (D) The expression of p65 in cytoplasm and nucleus of epithelial with or without SDF-1 stimulation analyzed by western blot. GAPDH and Histone H3 served as internal controls for cytoplasm and nucleus, respectively. $* \mathrm{P}<0.01$, $* * \mathrm{P}<0.05$ vs control. 


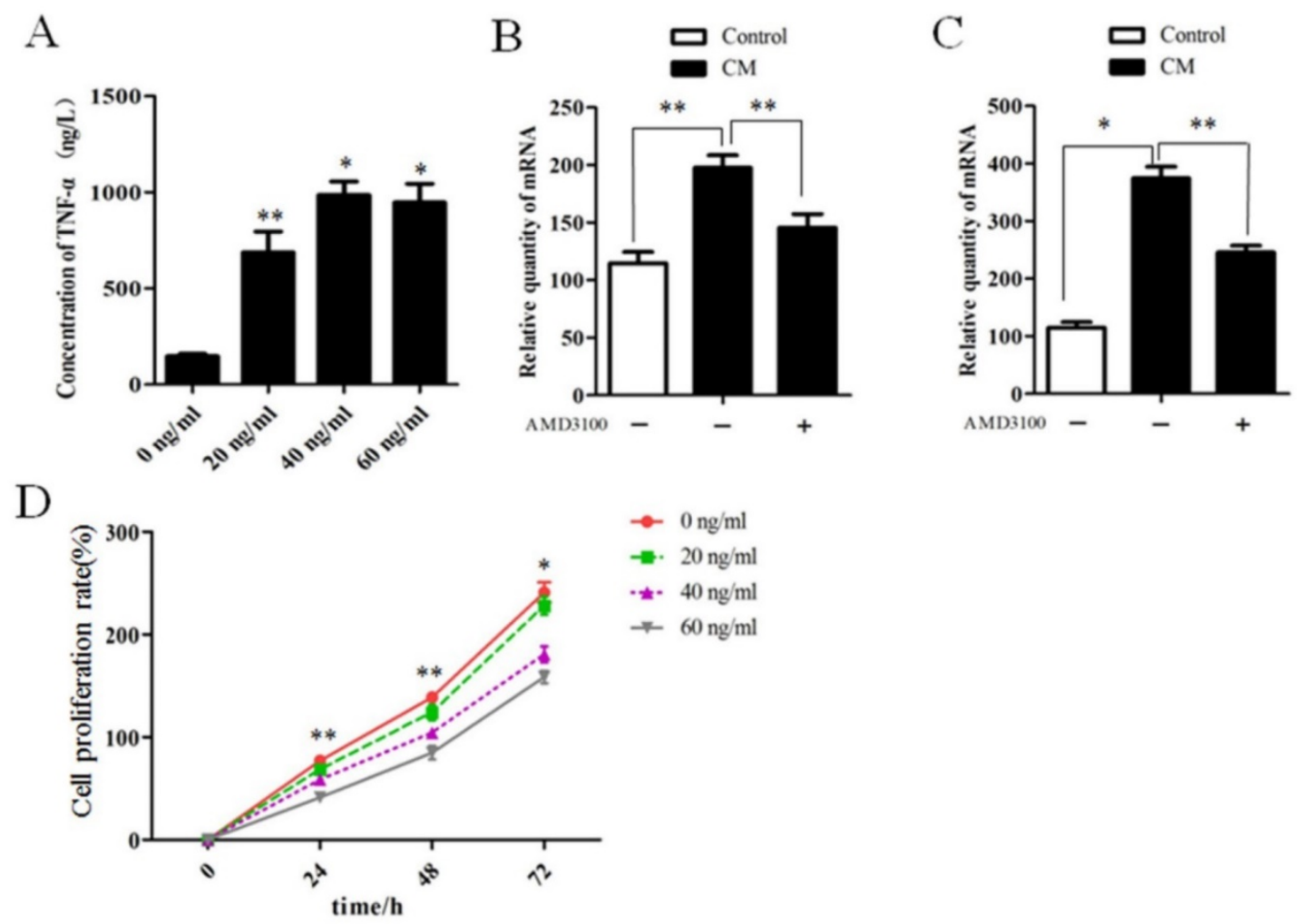

Figure 7. SDF-1 mediates LPS- and LTA-induced inflammatory response in epithelial cells and inhibits the proliferation of epithelial cells. (A) The concentration of TNF- $\alpha$ in medium secreted by epithelial cells stimulated by SDF- 1 at the concentration of $0,20,40,60 \mathrm{ng} / \mathrm{ml}$ measured by ELISA. *P<0.01, **P<0.05 vs control $(0 \mathrm{ng} / \mathrm{ml})$. (B and C) Epithelial cells with or without AMD3 100 pretreatment were cultured in conditioned medium (CM) from LPS-treated NFs or LTA-treated NFs for 3 days, and the secretion of TNF- $\alpha$ in the supernatant of culture was detected by ELISA. Epithelial cells cultured in MSM were used as control. Both LPS-treated NFs (B) and LTA-treated NFs (C) enhanced the section of TNF- $\alpha$ by epithelial cells compared with control. Pretreatment of epithelial cells with AMD3100 significantly attenuated the increase of TNF- $\alpha$. $* P<0.01$, $* * P<0.05$. (D) SDF-1 inhibited the proliferation of epithelial cells at the concentration of 40 and 60 $\mathrm{ng} / \mathrm{ml}$ at indicated time points compared with control $(0 \mathrm{ng} / \mathrm{ml}), * \mathrm{P}<0.01, * * \mathrm{P}<0.05 \mathrm{vs}$ control.

\section{SDF-1 induce inflammatory response in epithelial cells}

Fibroblast-secreted SDF-1 plays a key role in maintaining chronic inflammation [18, 19], and TNF-a is the earliest and primary endogenous mediator of inflammatory response [20]. For this reason, we tested whether SDF-1 could also directly stimulate pro-inflammatory response through evaluation of the secretion of TNF-a. Thus, we treated epithelial cells with SDF-1 in culture. Indeed, SDF-1 at the concentration of 20,40 , and $60 \mathrm{ng} / \mathrm{ml}$ promoted the secretion of TNF-a compared with untreated epithelial cells (Figure 7A).

Given the fact that LPS or LAT stimulated the secretion of SDF-1 by stromal fibroblasts (Figure 5), we further investigated whether inflammatory stromal fibroblasts induced by LPS or LTA could promote SDF-1-mediated inflammatory response in epithelial cells, we found that both LPS- and LTA-treated fibroblasts promoted TNF-a secretion by epithelial cells in a co-culture model, and AMD3100, a specific inhibitor of the receptor of SDF-1, alleviated the increased secretion of TNF-a by epithelial cells (Figure 7B and C).

\section{SDF-1 inhibit the proliferation of epithelial cells}

The effect of SDF-1 on epithelial cell proliferation was analyzed by using a CCK-8 kit. Results showed that on $24 \mathrm{~h}, 48 \mathrm{~h}$ and $12 \mathrm{~h}$, the proliferation rates of epithelial cells treated with SDF-1 at the concentration of 40 and $60 \mathrm{ng} / \mathrm{ml}$ was higher than untreated cells, but SDF-1 treatment at $20 \mathrm{ng} / \mathrm{ml}$ did not affect the proliferation ability of epithelial cells (Figure 7D).

\section{SDF-1 induce mastitis in a mouse model}

The initial finding that SDF-1 induced inflammatory response in epithelial cells in vitro suggested SDF-1 may function as a proinflammatory factor. We therefore wished to determine whether this ability of SDF-1 secreted by INFs could be attributed to a spread of inflammation from the infected alveoli to uninfected alveoli within the mammary gland. A mouse model was developed by teat injection of SDF-1. Compared with PBS-inoculated mice whose mammary tissue exhibited large luminal area, minimal cellular debris, and limited stromal area, and alveoli were characterized by polarized secretory cells with a large cytoplasmic: nuclear ratio (Figure 8A), we 
found that SDF-1 caused inflammation in the mammary gland of mice after injection for $24 \mathrm{~h}$, as indicated by a massive infiltration of leukocytes in the tissue of mice (Figure $8 \mathrm{~B}$ and $\mathrm{C}$ ). In addition, slight interstitial fibrosis was observed in mice treated by SDF-1 (Figure 8B). Pre-inoculation of AMD3100, a specific inhibitor of the receptor of SDF-1, by intraperitoneal injection inhibited SDF-1-induced mastitis in mice (Figure 8D).

\section{Discussion}

Considerable data exist describing the pathogenesis of bovine mastitis, for instance, evidence suggested that cellular components of neutrophils [21], macrophages [22], and lymphocytes [23], as well as epithelial cells in mammary gland [24] mediate the immune response during mastitis, however, the specific contributions of fibroblast, the predominant components of mammary stromal cells, to bovine mastitis have been unclear. Extending our previous finding that stromal fibroblasts derived from mammary gland of bovine with mastitis display inflammation-specific changes [15], we here initially showed that INFs extracted from bovine mammary glands with clinical mastitis had different expression pattern regarding to several ECM proteins, chemokines and cytokines compared to NFs from dairy cows during lactation. Organ fibrosis is usually characterized by exaggerated production of ECM [2], and an abnormal inflammatory response can lead to over-production and deposition of ECM components, leading to fibrosis. It can start during an inflammatory response in the damaged mammary gland tissue of bovine with mastitis [25]. Indeed, INFs exhibit increased collagen-1 and MMP1 expression, as well as increased SDF-1, IL-8, and CXCL2 secretion, both indicative of involvement of stromal fibroblasts in the process of inflammatory response and even progressive fibrosis if it was caused by the inflammation in bovine mammary gland. Many of proinflammatory cytokines induce the expression of growth factors, which directly stimulate the proliferation of fibroblasts, their differentiation into myofibroblasts, and the production of ECM [26]. We further demonstrated that the gram negative and gram-positive bacterial cell wall components LPS and LTA could make normal stromal fibroblast acquire these myofibroblastic phenotypes (Figure 2). Previous reports suggested that inflammatory epithelial cells induced by LPS and LTA also induced secretion of inflammatory mediators, such as TNF- $\alpha$, IL-6, IL-8, CXCL2, and CCL5, and expression of ECM protein in bovine stromal fibroblasts [16]. Based on this, we concluded that both inflammatory epithelial cells and virulence factors of bacteria that invaded into stroma may contribute to the acquisition of myofibroblastic phenotypes for stromal fibroblasts within mammary gland with mastitis.

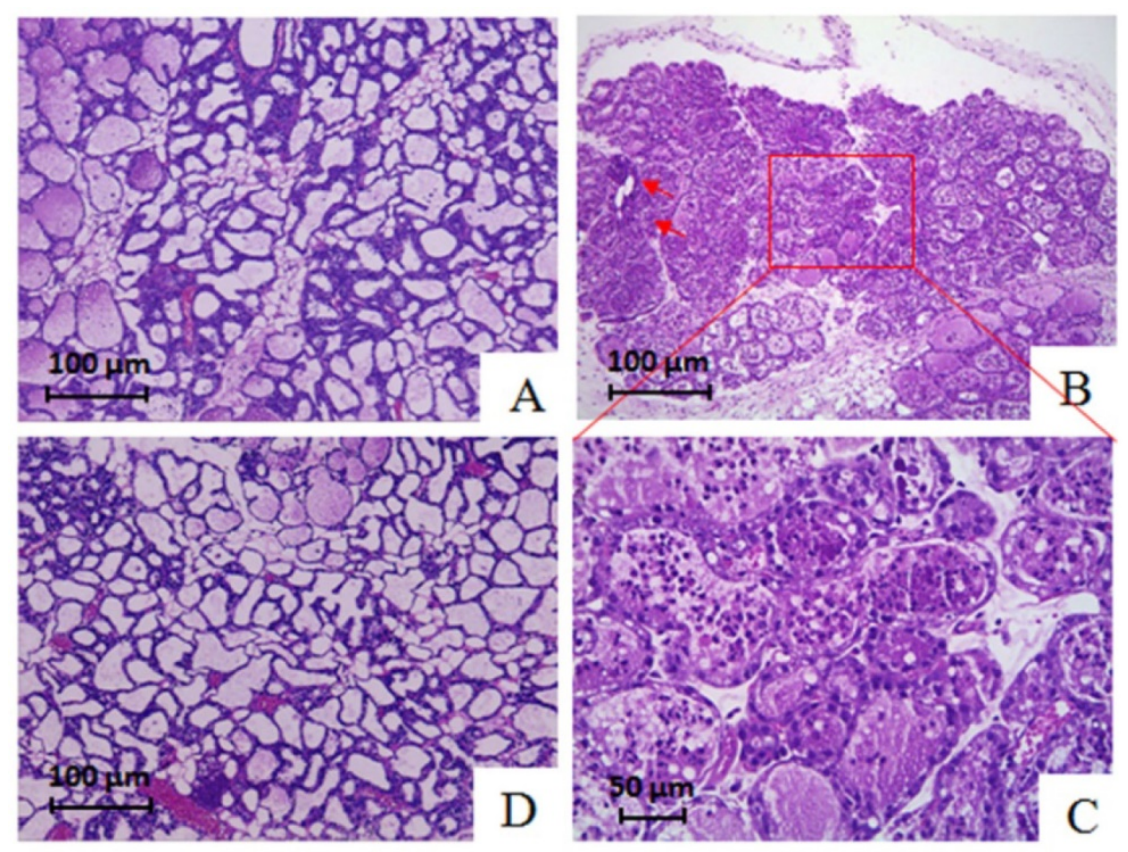

Figure 8. Representative hematoxylin and eosin staining sections of mammary tissue in mice. (A) Mammary parenchymal tissue of mice in control group exhibited large luminal area, minimal cellular debris, and limited stromal areas, and alveoli were characterized by polarized secretory cells with a large cytoplasmic: nuclear ratio. (B and C) Alveoli in an area of the gland of mice treated with SDF-1 were characterized by a massive infiltration of leukocytes in the lumen. The arrows indicated a slight interstitial fibrosis in tissue section of SDF-1-treated mice. (D) Pre-inoculation AMD3100 attenuated the infiltration of leukocytes in the lumen induced by SDF-1. 
Epithelial cells that undergo EMT is regarded as the potential sources of myofibroblasts in fibrosis of organs, including the kidney, liver, lung and heart [2]. EMT of epithelial cells occurs at an early stage of fibrosis of organs by the interaction with myofibroblasts [27]. Hallmarks of EMT include a decrease of cytokeratins, as well as a concomitant increase in mesenchymal markers such as a-SMA [28]. We have demonstrated that INFs induced EMT of epithelial cells in an indirect co-culture model. Although not all epithelial cells underwent morphological changes from cobblestone-like to spindle-like shape, upregulated a-SMA and downregulated cytokeratin did indicate EMT occurrence of epithelial cells induced by INFs. Fibroblasts can synthesize chemokines to regulate Inflammation as sentinel cells [29]. Fibroblasts play a key role in maintaining chronic inflammation by regulating the mobilization, recruitment, and immigration of leukocytes into inflammatory tissues through secretion of SDF-1 [18, 19]. SDF-1 could influence the EMT process of pancreatic cancer cells through non-canonical activation of Hedgehog pathway [30]. We found here SDF-1 was highly secreted/expressed by INFs (Figure 1), mastitic tissue (Figure 4), as well as LPS- or LTA-treated NFs (Figure 5) compared to their counterparts. Our work takes these other observations still further, by demonstrating that the process of EMT of epithelial cells was took place in the presence of exogenous SDF-1, as indicated by upregulated a-SMA and vimentin and downregulated cytokeratin expression.

Recent studies have identified that NF-KB was an important regulator of EMT in the majority of cell types [31-33]. NF-kB exists in an inactive form in the cytoplasm, and the treatment of cells with various inducers results in the degradation of IкB proteins via their phosphorylation and subsequent translocation of NF-kB p65 into the nucleus. In present study, epithelial cells treat with SDF-1 showed increased p-p65 and diverted p65 into the nucleus from cytoplasm. Based on these and our findings, we thus speculate that there is a cross-talk exists between SDF-1 and activation of NF-kB pathway, which is the critical for the INFs-induced EMT of epithelial cells.

The special binding of SDF-1 to a G-protein-coupled receptor, C-X-C chemokine receptor type 4 (CXCR4) has been found to be important for the process of cellular inflammatory, immune response, and blood homeostasis [34, 35]. AMD3100, a CXCR4 antagonist, which can inhibit binding of SDF-1 to CXCR4 and subsequent signal transduction, has been proposed that AMD3100 may also play an important role in treatment of many other SDF-1/CXCR4-regulated pathological processes. We found that SDF-1 induced significant inflammatory response both in vitro and in vivo experiments, and blocking CXCR4 by ADM3100 has a significant effect in attenuating the increase of TNF-a secretion in epithelial cells induced by LPS-NFs (Figure 7B) and LTA-NFs (Figure 7C) and alleviating inflammation reaction in alveoli of mouse mammary gland injected with SDF-1, which suggested that SDF-1 has an ability to induce inflammatory response of epithelial cells both in vitro and in vivo. Although INFs with increased SDF-1 secretion and exogenous SDF-1 stimulation induced the EMT occurrence of epithelial cells, we did not observe obvious tissue fibrosis in the mice injected with SDF-1, except for a slight fibrosis in a limited area, due to EMT occurring at an early stage of fibrosis [28].

In summary, we demonstrate that INFs extracted from bovine mammary glands with clinical mastitis had different expression pattern regarding to several ECM proteins, chemokines and cytokines compared to NFs from dairy cows during lactation. We conclude that stromal fibroblasts within mammary glands with mastitis contribute to EMT and inflammatory responses of epithelial cells through the secretion of SDF-1, which could result in the inflammation spread and fibrosis within mammary gland.

\section{Supplementary Material}

Supplementary Table S1.

http://www.ijbs.com/v13p0604s1.pdf

\section{Abbreviations}

INFs: Inflammation-associated fibroblasts

NFs: Normal fibroblasts

ECM: Extracellular matrix

EMT: Epithelial-mesenchymal transition

SDF-1: Stromal cell-derived factor 1

LPS: Lipopolysaccharide

LTA: Lipoteichoic acid

MSM: Minimum serum medium

PBS: Phosphate buffer saline

FBS: Fetal bovine serum

CCK-8: Cell Counting Kit-8

NF-CM: NF-conditioned medium

INF-CM: INF-conditioned medium

CM: Conditioned medium

\section{Acknowledgments}

The study was supported by the grants from National Natural Science Foundation of China (No.31402165).

\section{Competing Interests}

The authors have declared that no competing interest exists. 


\section{References}

1. Balkwill F. Cancer and the chemokine network. Nat Rev Cancer. 2004; 4: 540-50.

2. Zou XZ, Liu T, Gong ZC, Hu CP, Zhang Z. MicroRNAs-mediated epithelial-mesenchymal transition in fibrotic diseases. Eur J Pharmacol. 2017; 796: 190-206.

3. Ghosh AK, Quaggin SE, Vaughan DE. Molecular basis of organ fibrosis: potential therapeutic approaches. Exp Biol Med (Maywood). 2013; 238: 461-81.

4. Buckley CD. Why does chronic inflammation persist: An unexpected role for fibroblasts. Immunol Lett. 2011; 138: 12-4.

5. Buckley CD, Pilling D, Lord JM, Akbar AN, Scheel-Toellner D, Salmon M. Fibroblasts regulate the switch from acute resolving to chronic persistent inflammation. Trends Immunol. 2001; 22: 199-204.

6. Xu J, Mora A, Shim H, Stecenko A, Brigham KL, Rojas M. Role of the SDF-1/CXCR4 axis in the pathogenesis of lung injury and fibrosis. Am J Respir Cell Mol Biol. 2007; 37: 291-9.

7. Hogaboam CM, Steinhauser ML, Chensue SW, Kunkel SL. Novel roles for chemokines and fibroblasts in interstitial fibrosis. Kidney Int. 1998; 54: 2152-9.

8. Brouty-Boye D, Pottin-Clemenceau C, Doucet C, Jasmin C, Azzarone B. Chemokines and CD40 expression in human fibroblasts. Eur J Immunol. 2000; 30: 914-9.

9. Kobayashi $\mathrm{K}$, Sato $\mathrm{K}$, Kida $\mathrm{T}$, Omori $\mathrm{K}$, Hori $\mathrm{M}$, Ozaki $\mathrm{H}$, et al Stromal cell-derived factor-1alpha/C-X-C chemokine receptor type 4 axis promotes endothelial cell barrier integrity via phosphoinositide 3-kinase and Rac1 activation. Arterioscler Thromb Vasc Biol. 2014; 34: 1716-22.

10. Bleul CC, Fuhlbrigge RC, Casasnovas JM, Aiuti A, Springer TA. A highly efficacious lymphocyte chemoattractant, stromal cell-derived factor 1 (SDF-1). J Exp Med. 1996; 184: 1101-9.

11. Hattermann $K$, Holzenburg E, Hans F, Lucius R, Held-Feindt J, Mentlein R. Effects of the chemokine CXCL12 and combined internalization of its receptors CXCR4 and CXCR7 in human MCF-7 breast cancer cells. Cell Tissue Res. 2014; 357: $253-66$

12. Kang H, Watkins G, Parr C, Douglas-Jones A, Mansel RE, Jiang WG. Stromal cell derived factor-1: its influence on invasiveness and migration of breast cancer cells in vitro, and its association with prognosis and survival in human breast cancer. Breast Cancer Res. 2005; 7: R402-10.

13. Luker KE, Luker GD. Functions of CXCL12 and CXCR4 in breast cancer. Cancer Lett. 2006; 238: 30-41.

14. Zlotnik A. Chemokines and cancer. Int J Cancer. 2006; 119: 2026-9.

15. Chen Q, He G, Zhang W, Xu T, Qi H, Li J, et al. Stromal fibroblasts derived from mammary gland of bovine with mastitis display inflammation-specific changes. Sci Rep. 2016; 6: 27462

16. Zhang $\mathrm{W}$, Li X, Xu T, Ma M, Zhang $\mathrm{Y}$, Gao MQ. Inflammatory responses of stromal fibroblasts to inflammatory epithelial cells are involved in the pathogenesis of bovine mastitis. Exp Cell Res. 2016; 349: 45-52.

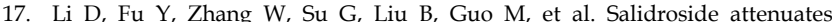
inflammatory responses by suppressing nuclear factor-kappaB and mitogen activated protein kinases activation in lipopolysaccharide-induced mastitis in mice. Inflamm Res. 2013; 62: 9-15.

18. Douglas MR, Morrison KE, Salmon M, Buckley CD. Why does inflammation persist: a dominant role for the stromal microenvironment? Expert Rev Mol Med. 2002; 4: 1-18

19. Suratt BT, Petty JM, Young SK, Malcolm KC, Lieber JG, Nick JA, et al. Role of the CXCR4/SDF-1 chemokine axis in circulating neutrophil homeostasis. Blood. 2004; 104: 565-71.

20. Chu X, Song K, Xu K, Zhang X, Zhang X, Song Y, et al. Ceftiofur attenuates lipopolysaccharide-induced acute lung injury. Int Immunopharmacol. 2010; 10: 600-4.

21. Sladek Z, Rysanek D, Ryznarova H, Faldyna M. Neutrophil apoptosis during experimentally induced Staphylococcus aureus mastitis. Vet Res. 2005; 36: $629-43$.

22. Sladek Z, Ryznarova H, Rysanek D. Macrophages of the bovine heifer mammary gland: morphological features during initiation and resolution of the inflammatory response. Anat Histol Embryol. 2006; 35: 116-24.

23. Sordillo LM, Streicher KL. Mammary gland immunity and mastitis susceptibility. J Mammary Gland Biol Neoplasia. 2002; 7: 135-46.

24. Schukken $\mathrm{YH}$, Gunther J, Fitzpatrick I, Fontaine MC, Goetze L, Holst O, et al. Host-response patterns of intramammary infections in dairy cows. Vet Immunol Immunopathol. 2011; 144: 270-89.

25. Benites NR, Guerra JL, Melville PA, da Costa EO. Aetiology and histopathology of bovine mastitis of espontaneous occurrence. J Vet Med B Infect Dis Vet Public Health. 2002; 49: 366-70.

26. Musters S, Coughlan K, McFadden T, Maple R, Mulvey T, Plaut K. Exogenous TGF-beta1 promotes stromal development in the heifer mammary gland. J Dairy Sci. 2004; 87: 896-904

27. Sakai N, Tager AM. Fibrosis of two: Epithelial cell-fibroblast interactions in pulmonary fibrosis. Biochim Biophys Acta. 2013; 1832: 911-21.

28. Zou XZ, Liu T, Gong ZC, Hu CP, Zhang Z. MicroRNAs-mediated epithelial-mesenchymal transition in fibrotic diseases. Eur J Pharmacol. 2016.

29. Smith RS, Smith TJ, Blieden TM, Phipps RP. Fibroblasts as sentinel cells. Synthesis of chemokines and regulation of inflammation. Am J Pathol. 1997; 151: 317-22.

30. Li X, Ma Q, Xu Q, Liu H, Lei J, Duan W, et al. SDF-1/CXCR4 signaling induces pancreatic cancer cell invasion and epithelial-mesenchymal transition in vitro through non-canonical activation of Hedgehog pathway. Cancer Lett. 2012; 322: 169-76.

31. Chua HL, Bhat-Nakshatri P, Clare SE, Morimiya A, Badve S, Nakshatri H. NF-kappaB represses E-cadherin expression and enhances epithelial to mesenchymal transition of mammary epithelial cells: potential involvement of ZEB-1 and ZEB-2. Oncogene. 2007; 26: 711-24.

32. Min C, Eddy SF, Sherr DH, Sonenshein GE. NF-kappaB and epithelial to mesenchymal transition of cancer. J Cell Biochem. 2008; 104: 733-44.

33. Shin SR, Sanchez-Velar N, Sherr DH, Sonenshein GE. 7,12-dimethylbenz(a)anthracene treatment of a c-rel mouse mammary tumor cell line induces epithelial to mesenchymal transition via activation of nuclear factor-kappaB. Cancer Res. 2006; 66: 2570-5.

34. Zlotnik A, Yoshie O. Chemokines: a new classification system and their role in immunity. Immunity. 2000; 12: 121-7.

35. D'Apuzzo M, Rolink A, Loetscher M, Hoxie JA, Clark-Lewis I, Melchers F, et al. The chemokine SDF-1, stromal cell-derived factor 1, attracts early stage B cell precursors via the chemokine receptor CXCR4. Eur J Immunol. 1997; 27: 1788-93. 